\title{
Colobomatous cupping of the optic disc
}

\author{
A. J. RINTOUL \\ Eye Unit, Royal Naval Hospital, Haslar, Gosport, Hants.
}

Coloboma confined to the optic disc is a relatively rare condition (Steinberg, I943), and has been described as unilateral in the majority of cases (Duke-Elder, 1964; François, 1964). However, manifestation of the deformity may occur in the other eye (Hughes, 1947), and a number of bilateral cases have now been described. This paper records another bilateral case.

\section{Case report}

A 35-year-old man was found to have defective vision in the right eye at a routine medical examination and was referred for ophthalmic examination. He gave a history of poor vision in the right eye since childhood, and of having first been seen by a doctor at the age of 23 when living in India. He was told that he had a "lazy eye" for which no treatment could be given. There was no previous history of ocular trauma and no relevant medical history. A family history was not available.

GENERAL PHYSICAL EXAMINATION

The patient was a fit young man of distinctly asthenic physique with arachnodactyly and low-set ears.

VISUAL AGUity

Right eye $6 / 36$ and $\mathrm{Nr}_{4}$, not improved (-I·75 D sph.)

Left eye $6 / 9$ and $\mathrm{N}_{5}$, not improved (-0.25 D sph.).

ANTERIOR SEGMENT

Posterior embryotoxon was present, most marked on the temporal aspect of both eyes, and the anterior chambers were of moderate depth. No abnormality was detected in either iris or lens and the vitreous was clear. The applanation tension was $18 \mathrm{~mm}$. $\mathrm{Hg}$ in each eye. The angles were open with a well-marked line of Schwalbe, corresponding to the embryotoxon, and numerous pectinate fibres.

\section{FUNDUS}

The right disc (Fig. I) was greater than average size with a small inferior conus and was deeply cupped, the lower half being glistening white. The upper half was of good colour with a sharp overhang which concealed the vessels at the disc margin.

The left disc (Fig. 2) was slightly greater than average size and deeply cupped over most of its area. The vessels emerged at the base of the cup through a nasally directed scleral canal, the temporal branches following the rim of the cup to make a steep exit from the temporal aspect. The nasal branches made a more direct exit by surmounting the overhanging edge of the cup on the nasal side.

No other fundus abnormality was found. 


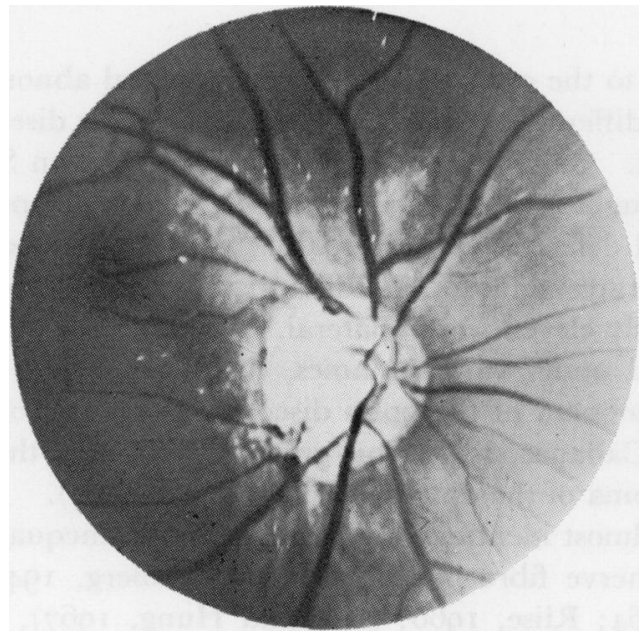

FIG. I Colobomatous cupping of right optic disc

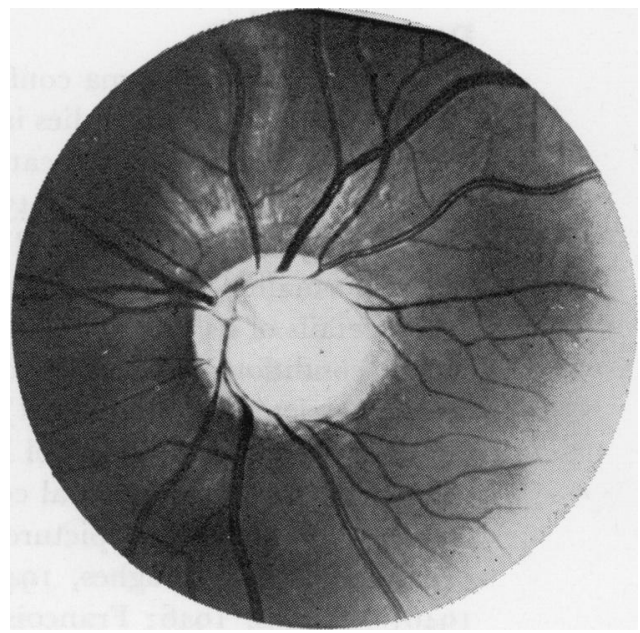

FIG. 2 Colobomatous cupping of left optic disc

VISUAL FIELDS

A dense nerve fibre bundle type of defect affected most of the upper half of the right visual field (Fig. 3) and an arcuate scotoma was delineated below the fixation point in the left (Fig. 4).

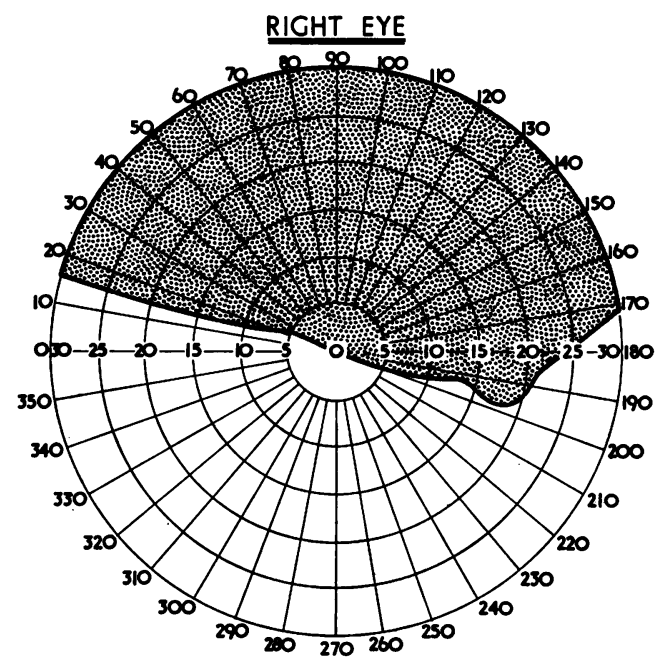

FIG. 3 Visual field defect. Target 2/1000 white. Visual acuity $6 / 3^{6}$

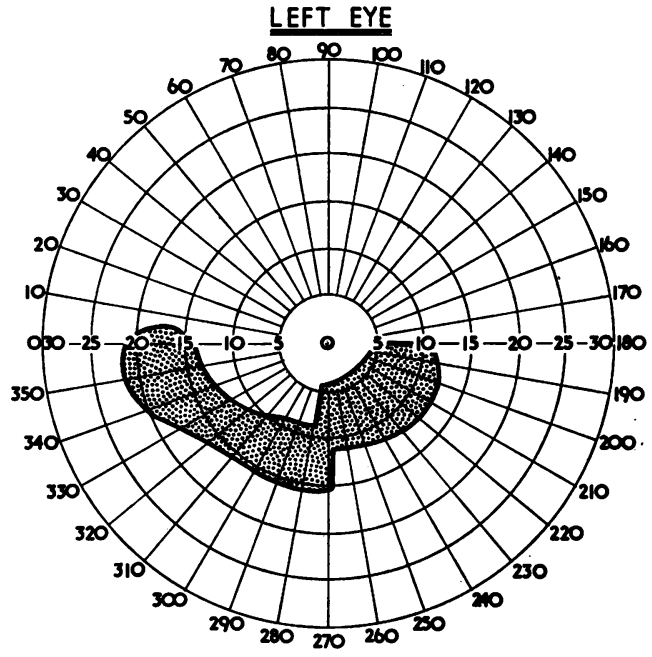

FIG. 4 Visual field defect. Target 2/1000 white. Visual acuity $6 / 9$

PROGRESS

The patient has been observed at intervals of 3 months over a period of 2 years, during which time the applanation tension has never been greater than $18 \mathrm{~mm}$. $\mathrm{Hg}$ in each eye and serial static perimetry has shown no change in the field defects.

A water drinking test produced a rise of $6 \mathrm{~mm}$. $\mathrm{Hg}$ in each eye after I hour with a return to the baseline after $90 \mathrm{~min}$.

DIAGNOSIS

Colobomatous cupping of the optic disc. 


\section{Discussion}

Bilateral typical coloboma confined to the optic disc is a developmental abnormality, the chief importance of which lies in its differentiation from progressive ocular diseases requiring early recognition and treatment. Such diseases include glaucoma (von Szily, I9o I ; Hillion, I9I I; Steinberg, I943; Hughes, I947; Gaipa, I96o), bilateral optic atrophy (Adler, I937), and bitemporal field defects (Rucker, 1946). Strictly defined as above, this abnormality is an exceedingly rare condition (Hillion, I9II). The literature provided details of 24 cases of which only eleven were bilateral.

The condition has been described under various names, coloboma of the optic nerve head (Terrien and Petit, I90 I), coloboma of the optic disc (Hird, I912), colobomatous cupping of the optic disc (Del Rio Cabanas, 1949), low-grade coloboma of the optic disc (Henkind, I 963), and partial coloboma of the optic nerve (François, 1964).

The clear-cut clinical picture is almost identical in every case, with unequal visual loss (Steinberg, I943; Hughes, I947), nerve fibre bundle defect (Steinberg, 1943; Rucker, I946; Magnus, I946; François, I964; Riise, I966; Kim and Hung, I967), the typical appearance of the optic disc, and the arrangement of the vessels adequately described by Caspar (r887). There is usually no other abnormality in the fundus.

\section{AETIOLOGY}

This condition has been well documented clinically (Steinberg, r 943; Del Rio Cabañas, I949; Offret, I 967) and experimentally (Nicholls and Tansley, r938; Payne, I 94 I). The inferior situation of cup in all cases described suggests the distal extremity of the embryonic fissure as the likely site of the abnormality. Closure of the embryonic fissure begins at the I I $\mathrm{mm}$. stage and is normally complete by the I5 mm. stage (Offret, I967). Some controversy exists over which germinal layer is initially at fault, ectoderm (Offret, I 967) or mesoderm (Calhoun, 1930). However, most authorities are agreed that the origin of the defect lies in excessive proliferation of the inner layer of the optic cup. This is simply an exaggeration of the normal mechanism of closure. Since the first sign of orientation of the paraxial mesoderm does not appear until the $18 \mathrm{~mm}$. stage (Pedler, I96 I), abnormal ingrowth of mesoderm can play only a sequential role in causation (Steinberg, 1943). The inheritance of typical coloboma as an irregular dominant with varying expressivity and greater than 30 per cent. penetrance is well established (Duke-Elder, 1964).

ASSOGIATED DEFECTS

Arachnodactyly (Curtius, I959), low-slung ears (Manara, I959), and mandibular dysostosis (François and Haustrate, I 954) have been described in association with typical coloboma. No previous mention of posterior embryotoxon has been found in association with colobomatous cupping, although Kim and Hung (1967) described "limbal leucomas". The presence of a myopic error of refraction has been reported in most of the cases on record (Terrien and Petit, I90 I Hillion, I9 I ; Rucker, I946; Hughes, I947; Caccamise, I 954; Kruse and Utermann, 1963; Ernyei, I964; François, 1964; Kipiotis and Theodosiadis, I964; Riise, I 966).

DIFFERENTIAL DIAGNOSIS

Hypoplasia of the optic nerve can always be distinguished by the small size of the optic disc and is often accompanied by radiological evidence of a small optic canal on the same side (Ewald, i 967 ).

The myopic crescent is commonly temporal and progressive, and may be accompanied by other signs in the fundus (Caccamise, 1954; François, 1964). 
Differentiation of colobomatous cupping from glaucomatous cupping may present problems, especially when accompanied by nerve fibre bundle defects (Hillion, I9I I; Magnus, 1946; Hughes, 1947). Amblyopia is always present from an early age and the typical colobomatous cup is deepest in the lower half of the disc, resulting in a corresponding field defect which is non-progressive. Provocative tests to detect the presence of embarrassed outflow are negative (Magnus, 1946). The presence of coloboma results in the unusual arrangement of vessels on the disc, already noted.

Adler (1937) and Rucker (1946) have reported cases initially diagnosed as pituitary tumour, and Caccamise (1954) drew attention to the presence of a bitemporal field defect in his case.

\section{Summary}

A case of bilateral colobomatous cupping of the optic disc, with field defect, and associated abnormality is presented. Attention is drawn to the importance of differentiating this condition from progressive ocular disease requiring treatment.

I thank Surgeon Captain T. C. Barras, Royal Navy, for his help in the preparation of this paper.

\section{References}

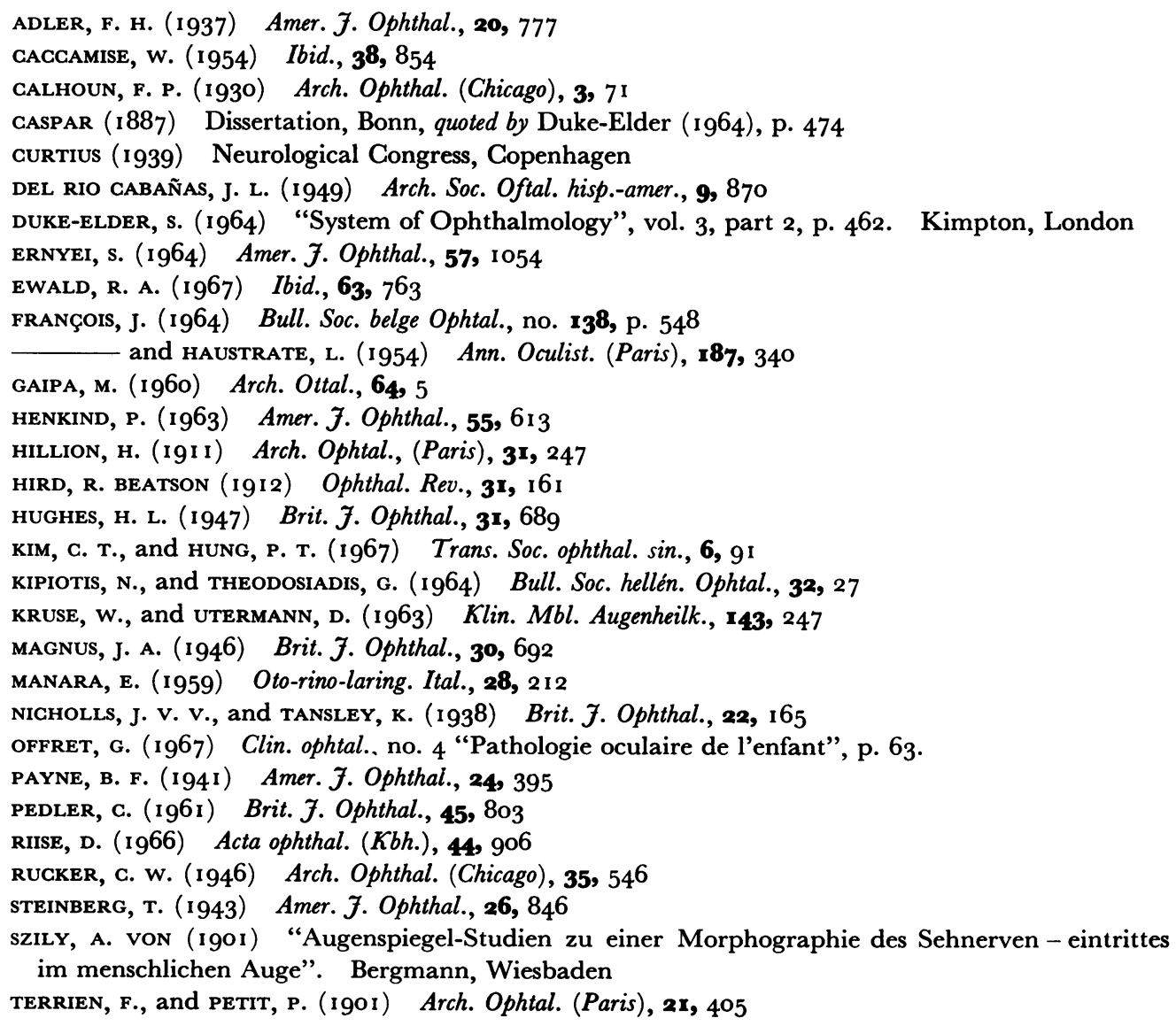

